26. Jahrestagung der Arbeitsgemeinschaft Dermatologische Histologie (ADH)

11.-13. Mai 2018

Mannheim, Deutschland

www.adh-kongress.de

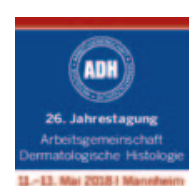

Senologiekongress - 38. Jahrestagung der Deutschen Gesellschaft für Senologie

14.-16. Juni 2018

Stuttgart, Deutschland

www.senologiekongress.de
12. Jahrestagung der Mitteldeutschen Gesellschaft für Frauenheilkunde und Geburtshilfe

25.-26. Mai 2018

Magdeburg, Deutschland

www.mgfg-kongress.de

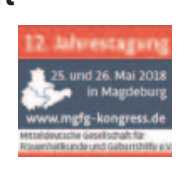

DEGRO - 24. Jahrestagung der Deutschen Gesellschaft für Radioonkologie

21.-24. Juni 2018

Leipzig, Deutschland

www.degro-jahrestagung.de

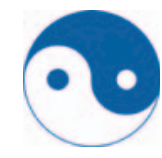

GI-Oncology 2018 - 14. Interdisziplinäres Update 09. Juni 2018

Wiesbaden, Deutschland www.gi-oncology.de

\section{G1-ONCOLOGY 2018} -14. Interdisziplinaros Updato

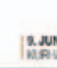

\section{BNGO-Hauptstadtkongress}

22.-23. Juni 2018

Berlin, Deutschland

www.bngo-kongress.de
APRIL

Leitlinie Kompakt: Kolorektales Karzinom

- Fokus: Metastasiertes Kolonkarzinom

19. April 2018

Online-Seminar

www.dgho-service.de $\rightarrow$ Webinare

\section{Gyn-Onko Update}

20.-21. April 2018

Mainz, Deutschland

https://gyn-onko-update.com

MAI

9. Akademie Knochen und Krebs

04.-05. Mai 2018

München, Deutschland

www.akuk-anmeldung.de

WPO Vertiefungsseminar 4: Systemische Familienmedizin - Therapie und Beratung von Krebskranken und Angehörigen

04.-05. Mai 2018

Freiburg im Breisgau, Deutschland www.wpo-ev.de

\section{Excellence in Oncology - Kolorektales} Karzinom

05. Mai 2018

Stuttgart, Deutschland

www.excellence-oncology.com/

kolorektales-karzinom
Excellence in Oncology - Lungenkarzinom 05. Mai 2018

Berlin, Deutschland

www.excellence-oncology.com/lungenkarzinom

EAPC - 10th World Research Congress of the European Association for Palliative Care

24.-26. Mai 2018

Bern, Schweiz

www.eapcnet.eu

JUNI

European Gastric Cancer Congress (EGCC) 07.-08. Juni 2018

Leiden, Niederlande

www.egcc.org

WPO Vertiefungsseminar 5: Trauer und Abschied in der Beratung und Therapie für Angehörige von Krebspatienten 08.-09. Juni 2018

Hamburg, Deutschland

www.wpo-ev.de

Onko Drei Länder Neues zu Immunonkologika und gezielten Therapien 15.-16. Juni 2018

München, Deutschland www.fomf.de $\rightarrow$ CME-Fortbildungen
4. Interdisziplinäres Symposium "Innovations in Oncology" 22. Juni 20128

Berlin, Deutschland www.nct-heidelberg.de $\rightarrow$ Für Ärzte $\rightarrow$ Veranstaltungen

WPO Vertiefungsseminar 6:

Gruppentherapie bei Krebspatienten mit Progredienzangst

22.-23. Juni 2018

Hamburg, Deutschland

www.wpo-ev.de

15. Trainingskurs für Klinische Infektiologie in der Hämatologie und Onkologie

29.-30. Juni 2018

Berlin, Deutschland

www.dgho-service.de $\rightarrow$ Veranstaltungen

Update Hämatologie/Onkologie 2018 29.-30. Juni 2018

Bonn, Deutschland

www.dgho-service.de $\rightarrow$ Veranstaltungen

\title{
KARGER
}

() 2018 S. Karger GmbH, Freiburg 